\title{
Research on Rapid Measurement of Methanol in Immobilized Lipase Synthesis of Biodiesel Based on Near Infrared Technology
}

\author{
Ji Li ${ }^{1}$, Jian Xing ${ }^{1 *}$, Guohua Chen ${ }^{1}$, Senshen $\mathrm{Yu}^{2}$ \\ 1. College of Mechanical and Electrical Engineering, Beijing University of Chemical Technology \\ 2. College of Life Science and Technology, Beijing University of Chemical Technology \\ Beijing, China \\ xingjian@mail.buct.edu.cn
}

\begin{abstract}
Aiming at the measurement of methanol content during the synthesis of methyl oleate from methanol and oleic acid with immobilized lipase in solvent-free and non aqueous media, a rapid measurement method of "centrifugation-near infrared(NIR) transmission" was proposed and a portable NIR transmission measurement system for methanol content was designed. The linear relationship between the methanol content and the absorbance of solution based on Lambert-Beer law was established. The experimental results demonstrate that this measurement system is stable and reliable with methanol content in actual work range of $0 \sim 25 \%$, the root mean square error (RMSE) between the measured value and real value is $\mathbf{1 . 0 3 6}$, and the relative standard deviation (RSD) of the sample data is $1.06 \% \sim 7.58 \%$. A mathematical model was established with partial least squares (PLS) in the spectral range of $4500 \sim 9000 \mathrm{~cm}^{-}$ 1, six groups of samples with different methanol content were measured and the RMSE between the measured value of this system and the PLS model prediction is 0.263 . It provides an effective measurement technology which contributes to increase the conversion and efficiency in biodiesel synthesis.
\end{abstract}

Keywords-methanol content; near infrared technology; rapid measurement; biodiesel; immobilized lipase

\section{INTRODUCTION}

Biodiesel is a kind of fatty acid ester produced by animal and vegetable oil and short-chain alcohols such as methanol through esterification or transesterification under catalytic conditions. Biodiesel has excellent features such as renewable, environment-friendly, good combustion performance and biodegradability that the traditional petrochemical energy cannot match, is a typical "green energy" [1 2].

The current synthesis methods of biodiesel mainly include chemical catalysis (acid or base as catalysts), bio-enzyme catalysis, engineering microalgae method and supercritical method [3]. The bio-enzyme synthesis of biodiesel has the advantages of milder reaction conditions, less alcohol consumption, no pollution in the reaction process and the reaction product could be collected and purified easily compared with traditional chemical methods [4]. In the process of bio-enzyme synthesis of biodiesel, too low concentration of methanol in system is not conducive to the forward reaction, but too high concentration of methanol in the reaction system will strongly inhibit the activity of the enzyme and reduce the reaction conversion [5 6]. The main factors that restrict the development of immobilized lipase synthesis of biodiesel are the inhibitory effect of methanol on lipase and higher cost of lipase [7]. This condition can be improved to some extent by batch added or methanol feeding, but it still can't reflect the influence of methanol in the system during the synthesis of biodiesel directly. Therefore, in order to ensure the catalytic efficiency of lipase, increase the reaction conversion and reuse rate of lipase and reduce the production cost, it is vital to research and develop an accurate, convenient and rapid measurement method to monitor and control the methanol content during the synthesis of biodiesel, which is of great significance to the industrialization of biodiesel [8].

Traditional methods for the measurement of methanol content include colorimetry, gas chromatography (GC) and high performance liquid chromatography (HPLC) [9]. Wang et al. [10] chose the $590 \mathrm{~nm}$ wavelength light source as the measurement wavelength and measured the methanol content in liquor by magenta-sulfurous acid spectrophotometry, the oxidation time was $15 \mathrm{~min}$ and the coloration time was $40 \mathrm{~min}$, the linearity of the established standard curve was rather good and the relative standard deviation (RSD) was less than $5 \%$. Li et al. [11] used headspace gas chromatography (HS-GC) to research the best measurement conditions of biodiesel at different storage times from the aspects of chromatographic column, chromatographic conditions and the choice of headspace sampling conditions, achieved the measurement of trace methanol in biodiesel. Paraschivescu et al. [12] proposed a headspace solid-phase microextraction method for the measurement of methanol content in biodiesel, using polydimethylsiloxane solid-phase microextraction for fiber sampling, and then analyzing the samples by gas chromatography, this method can measure extremely low methanol content with good repeatability and recovery. All the above methods generally have the disadvantages of complicated detection process, high cost, long analysis time and need for professional operation, cannot meet the requirements of on-site rapid analysis.

Near infrared (NIR) spectroscopy technology enables the qualitative and quantitative analysis of the components of the samples by detecting the vibration absorption of the frequency doubling and combined frequency between atoms in organic molecules. It has the advantages of short detection cycle, low cost, no need for sample pretreatment or only need simple pretreatment and no pollution to the environment, widely used 
in petrochemical industry, agriculture, food science, medicine and life science etc. [13 14]. At present, the NIR spectroscopy technology provides good application effects in petrochemical industry, but there are not much relevant research data on process analytical technology (PAT) in synthesis of biodiesel. According to the rapid measurement of methanol content during immobilized lipase synthesis of biodiesel, a NIR transmission measurement system of methanol content was designed according to the one time frequency vibration absorption characteristic peak of methanol. The specific infrared $1550 \mathrm{~nm}$ spectrum was used as the incident light source and a linear relationship between the methanol content and the absorbance of solution was established, which provides an effective reference method for the rapid measurement of methanol content during the immobilized lipase synthesis of biodiesel.

\section{MEASUREMENT PRINCIPLE AND SYSTEM IMPLEMENTATION}

\section{A. The Measurement Principle}

\section{(1) Beer-Lambert Law}

NIR spectroscopy measurement methods mainly include transmission, diffuse reflectance and diffuse transmission, an appropriate method should be selected according to different analysis objectives and the external characteristics of the samples in actual analysis. The transmission measurements are often used for homogeneous or transparent liquid samples.

There is no particulate matter that can scatter the light source in the pure solution system, the optical path and the sample concentration are in accord with Beer-Lambert law, that is, if the liquid layer thickness is certain, the absorption intensity of the material in a certain wavelength light source is proportional to the concentration of the light-absorbing material, there exists the following equation:

$$
A=\lg \frac{I_{0}}{I}=\varepsilon b c
$$

Where $A$ is the absorbance, $I_{0}$ is the incident light intensity, $I$ is the transmitted light intensity, $\varepsilon$ is the solution absorptivity, $b$ is the liquid layer thickness (i.e., the optical path), $c$ is the solution concentration.

\section{(2) Wavelength Selection}

In the non aqueous catalytic synthesis of biodiesel by immobilized lipase, 1 mol oleic acid and 1 mol methanol are enzymatically catalyzed to produce 1 mol methyl oleate and 1 mol water, the chemical reaction equation can be described as:

$$
\mathrm{C}_{17} \mathrm{H}_{33} \mathrm{COOH}+\mathrm{CH}_{3} \mathrm{OH} \rightleftharpoons \text { Lipase } \mathrm{C}_{17} \mathrm{H}_{33} \mathrm{COOCH}_{3}+\mathrm{H}_{2} \mathrm{O}(2)
$$

It is a reversible reaction, the excessive water in this system will inhibit the forward reaction and destroy the microenvironment of the lipase, as a result the stability and activity of the lipase are decreased. Actually, a sufficient amount of water absorbent (molecular sieve, silica gel, etc.) should be added in the reaction system to remove the excess water in time. Since the lipase itself is insoluble in most organic solvents, and in the two kinds of commonly used water absorbents, the main component of the molecular sieve is aluminosilicate and the silica gel is silica, both are not easily soluble in organic solvents. So, the upper liquid phase contains three components oleic acid, methanol and methyl oleate after the centrifugation of the reaction solution to precipitate solid particles. In fact, it is not absolutely anhydrous in non aqueous systems, catalytic enzyme needs a small amount of water bound to it to maintain the conformation and function itself, this part of water is hydrated with the enzyme powder and combined with the immobilized carrier and is insoluble in the less polar oleic acid and methyl oleate in this reaction system. Generally, the water content of a typical non aqueous enzyme system is less than $0.01 \%$ [15]. It can be seen that the presence of trace amounts of bound water does not affect the measurement of methanol content in this reaction system. Therefore, we measure the methanol content in the threecomponent system containing oleic acid, methanol and methyl oleate in the upper liquid phase to reflect the inhibitory effect of methanol on lipase.

According to equation (2), the three organic compounds oleic acid, methanol, and methyl oleate contain three chemical groups $\mathrm{C}-\mathrm{H}, \mathrm{O}-\mathrm{H}$, and $\mathrm{C}=\mathrm{O}, \mathrm{O}-\mathrm{H}$ exists in two different chemical environments of carboxylic acid and alcohol with different characteristic absorption bands. The optimum wavelength for measuring the methanol content should be near the wavelength where $\mathrm{O}-\mathrm{H}$ in the alcohol absorbs most strongly, and other chemical groups present in the solution absorb weakly at this wavelength. In fact, the multi-class double frequency vibration absorption of the chemical groups is very weak, usually masked by the one time frequency or the frequency-doubled. The $\mathrm{O}-\mathrm{H}$ in alcohol has a strong one time frequency vibration absorption in the range of $1520 \sim 1639 \mathrm{~nm}$, so the $1550 \mathrm{~nm}$ NIR light source was selected to measure the methanol content of the three-component mixed solution system. The vibration absorption bands of the three chemical groups are shown in Table 1.

TABLE I. ABSORPTION BANDS OF C-H, C=O, O-H IN ORGANIC COMPOUNDS(NM)

\begin{tabular}{ccccc}
\hline \multirow{2}{*}{$\begin{array}{c}\text { Vibration } \\
\text { Types }\end{array}$} & C-H & C=O & \multicolumn{2}{c}{ O-H } \\
\cline { 4 - 6 } & & 2250 & $\begin{array}{c}\text { Carboxylic } \\
\text { acid }\end{array}$ & Alcohols \\
\hline $\begin{array}{c}\text { combined } \\
\text { frequency } \\
\text { one time } \\
\text { frequency } \\
\text { frequency- } \\
\text { doubled } \\
\text { third }\end{array}$ & $1690 \sim 1755$ & $2615 \sim 3548$ & $1699-1833$ & $1520 \sim 1639$ \\
harmonic & $845 \sim 878$ & $1333 \sim 1935$ & $866 \sim 999$ & $775 \sim 894$ \\
\hline
\end{tabular}

\section{B. Implementation of The Measurement System}

The light source of the solution absorbance measurement system is a $1550 \mathrm{~nm}$ distributed feedback (DFB) laser diode, the detector is an InGaAs photodetector with a spectral response in $900 \sim 1700 \mathrm{~nm}$ wavelength range and the responsivity at 1550 $\mathrm{nm}$ of the detector is $0.9 \mathrm{~mA} / \mathrm{mW}$, the sample cell of this measurement system is a $5 \mathrm{~mm}$ quartz cuvette.

In the process of sample measurement, the parallel light emitted by the continuous and constant laser diode is transmitted through the sample cell and then absorbed by the 
detector. The detector outputs current signal under a certain light intensity, this current signal is converted to a high enough digital voltage signal after I/V conversion, amplification, and signal acquisition. Then, the digital voltage signal is transmitted to the host computer through serial communication, and the host computer stores and displays the voltage value after average filtering, the workflow of the measurement system is shown in Figure 1.

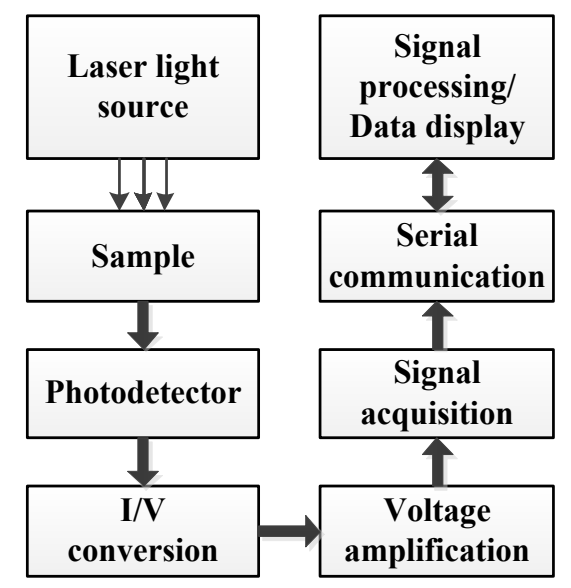

Fig. 1. Workflow of the measurement system

In this measurement system, the voltage output of the empty cuvette collected is used as a reference. According to equation (1), it can be deduced that the solution absorbance value calculation formula is:

$$
A=\lg \frac{I_{0}}{I}=\lg \frac{V_{0}}{V}
$$

Where $V_{0}$ is the voltage output value of the system to detect the empty cuvette, $V$ is the voltage output value of the system to detect the solution sample. According to formula (3), the absorbance of different solution samples was calculated and the linear relationship between the methanol content and the absorbance of the samples was established.

\section{EXPERIMENTAL VERIFICATIONN AND DATA ANALYSIS}

\section{A. The Major Instruments and Reagents}

Antaris II FT-NIR spectrometer with RESULT 3.0 spectral collecting software and TQ Analyst 8.0 spectral analyzing software (Thermo Fisher Scientific Inc., USA), constant temperature shaking table (Harbin Donglian Electronics Co., Ltd., China), electronic balance (Shanghai Zhuojing Electronic Technology Co., Ltd., China), vortex oscillator (Scientific Industries Inc., USA) and $50 \mathrm{ml}$ high speed centrifuge (Shanghai Luxiangyi Instrument Co., Ltd., China).

Analytically pure methyl oleate was purchased from Tianjin Guangfu Fine Chemical Research Institute (Tianjin, China), analytically pure oleic acid and anhydrous methanol were purchased from Guangdong Xilong Scientific Co., Ltd. (Guangdong, China), Novozyme 435 immobilized lipase was purchased from Novozymes (China) Biotechnology Co., Ltd. (Tianjin, China), 3A molecular sieve, silica gel and other chemicals were obtained from commercial sources.

\section{B. Spectral Acquisition and Analysis}

The NIR spectra of oleic acid, methanol and methyl oleate were measured in the spectral range of $4000 \sim 10000 \mathrm{~cm}^{-1}$ using the Antaris II FT-NIR spectrometer, after preheating for 30 minutes, the transmission accessory module and a $5 \mathrm{~mm}$ quartz cuvette were used for spectral acquisition. Selecting the built-in empty light path as a reference, the scan number is 32 , the spectral resolution is $4 \mathrm{~cm}^{-1}$, the gain is $1 \mathrm{X}$, the NIR spectrum of the three reagents is shown in Figure 2.

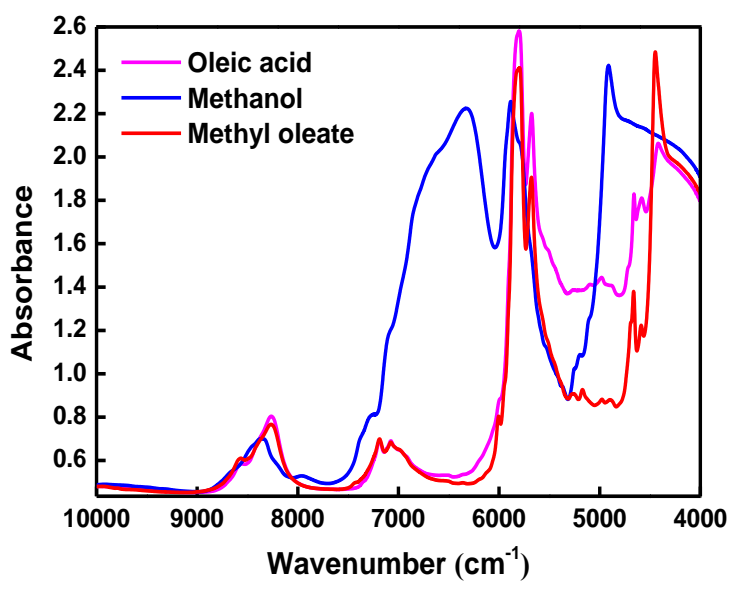

Fig. 2. The raw NIR spectra of the three reagments

It can be seen from Figure 2 that there is a broad absorption band in the range of $6050 \sim 7000 \mathrm{~cm}^{-1}$ in the methanol spectrum, which is the strong one time frequency vibration absorption of the $\mathrm{O}-\mathrm{H}$ hydrogen bonds of methanol in this spectral range, the absorption peak of this band is near $6300 \mathrm{~cm}^{-1}$. Since the similar chemical structure of oleic acid and methyl oleate, both of their spectrum mainly contain the vibration absorption of frequency doubling and combined frequency of $\mathrm{C}-\mathrm{H}$ bond, there is no obvious significant characteristic absorption peak and the absorbance is close to the baseline of the spectrum in the range of $6200 \sim 6800 \mathrm{~cm}^{-1}$. The absorbance of oleic acid and methyl oleate was similar in the range of $6400 \sim 7000 \mathrm{~cm}^{-1}$.

\section{The Standard Curve of Methonal Content}

During enzymatic synthesis of biodiesel, the methods of adding methanol include one-time addition, step-by-step addition and feeding addition. The molar ratio of methanol to oleic acid is generally not more than $3: 1$ in actual reaction, according to this ratio, the methanol content studied here is $0 \%-25 \%$.

Nine mixture samples of oleic acid, methanol and methyl oleate were prepared, in which the volume ratio of oleic acid to methyl oleate is $1: 1$ and the methanol content is $0 \% \sim 25 \%$, each sample was shaken up in a $5 \mathrm{ml}$ centrifuge tube, the absorbance values of samples prepared with different concentrations of methanol are shown in Table 2. 
TABLE II. ABSORBANCE OF NINE METHANOL CONCENTRATION SAMPLES

\begin{tabular}{ccccc}
\hline \multirow{4}{*}{ No. } & \multicolumn{3}{c}{ Content \%(v/v) } & \\
\cline { 2 - 4 } & Methanol & Oleic acid & Methyl oleate & \\
\hline 1 & 0 & 50 & 50 & 0.036 \\
2 & 2 & 49 & 49 & 0.068 \\
3 & 4 & 48 & 48 & 0.085 \\
4 & 6 & 47 & 47 & 0.109 \\
5 & 8 & 46 & 46 & 0.134 \\
6 & 10 & 45 & 45 & 0.165 \\
7 & 15 & 42.5 & 42.5 & 0.243 \\
8 & 20 & 40 & 40 & 0.335 \\
9 & 25 & 37.5 & 37.5 & 0.413 \\
\hline
\end{tabular}

Taking the methanol content as the abscissa and the solution absorbance as the ordinate, the standard curve formula of methanol content was obtained by applying the least squares method with the nine experimental data in Table 2:

$$
y=0.0152 x+0.0248
$$

The coefficient of determination $r^{2}$ is 0.9937 , and the fitting curve of methanol content is shown in Figure 3.

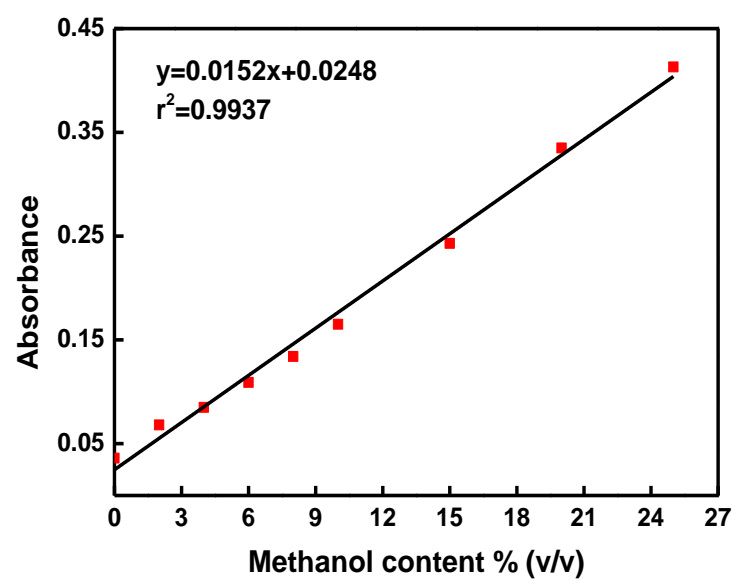

Fig. 3. The methanol content fitting curve

\section{Influence of the solubility of lipase and water absorbent at different ratios of oleic acid and methyl oleate}

The effects of solid particles (lipase and water absorbent) and different ratios of oleic acid and methyl oleate on the measurement of methanol content were discussed. $25 \mathrm{~g}$ oleic acid, $25 \mathrm{~g}$ methanol and $25 \mathrm{~g}$ methyl oleate were added in sequence to three $50 \mathrm{ml}$ shake flasks. According to the maximum addition amount of lipase and water absorbent (10\%wt lipase, $15 \%$ wt water absorbent) under the optimal reaction conditions as described in the literature [16 17], take the Novozyme 435 immobilized lipase, 3A molecular sieve, and silica gel water absorbent commonly used in synthesis biodiesel as the research object. $2.5 \mathrm{~g}$ lipase, $3.75 \mathrm{~g}$ molecular sieve and $3.75 \mathrm{~g}$ silica gel were added to each shake flask, the operation conditions of shaking table were $40^{\circ} \mathrm{C}$ and $200 \mathrm{rpm}$. After confined shaking for $4 \mathrm{~h}$, the three reagents were transferred to $50 \mathrm{ml}$ centrifuge tubes, and the solid particles were precipitated by centrifugation at $8000 \mathrm{~g}$ for 2 minutes. The supernatants of the three solutions obtained by the above treatments were collected. Ten samples of three-component solution with different methanol content after centrifugation were prepared, the content of the three reagents was guaranteed to conform to the actual reaction conditions as far as possible in the range of methanol content studied in this paper. Samples of different methanol content are shown in Table 3.

TABLE III. MEASUREMENT RESULTS OF TEN SAMPLES OF METHANOL $(\mathrm{N}=5)$

\begin{tabular}{ccccc}
\hline \multirow{4}{*}{ No. } & \multicolumn{3}{c}{ Content \%(v/v) } & RSD\% \\
\cline { 2 - 4 } & Methanol & Oleic acid & Methyl oleate & \\
\hline 1 & 1 & 13 & 86 & 7.58 \\
2 & 3 & 25 & 72 & 3.49 \\
3 & 7 & 35 & 58 & 1.61 \\
4 & 9 & 42 & 49 & 2.20 \\
5 & 12 & 66 & 22 & 1.10 \\
6 & 14 & 22 & 64 & 1.62 \\
7 & 16 & 84 & 0 & 1.29 \\
8 & 19 & 10 & 71 & 1.18 \\
9 & 22 & 60 & 18 & 1.06 \\
10 & 25 & 15 & 60 & 1.28 \\
\hline
\end{tabular}

According to formula (4), the relationship between the methanol content and the absorbance value of solution is:

$$
x=\frac{y-0.0248}{0.0152}
$$

The root mean square error (RMSE) between the measurement value and the real value of the methanol content obtained from formula (5) is 1.036 . The RSD of the results of five measurements per sample is $1.06 \% \sim 7.58 \%$ as shown in Table 3, which satisfied the measurement stability requirements of the system. The scatter plots of the measurement of methanol content are shown in Figure 4.

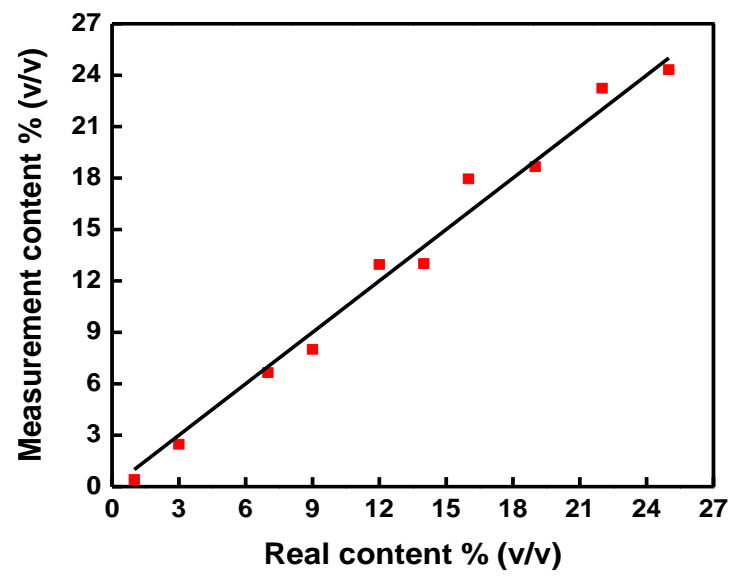

Fig. 4. Scatter plots of the measurement of methanol content 


\section{E. Accuracy comparison}

According to the proportions of the first six groups in Table 2 , six samples with $0 \% \sim 10 \%$ methanol content were prepared. The NIR spectroscopy data of these samples were collected using the Antaris II FT-NIR spectrometer at the same measurement conditions as Figure 2. According to the characteristics of the spectral changes of the three reagents in Figure 2, the spectral information of the three reagents varies widely in the spectral range of $4500 \sim 9000 \mathrm{~cm}^{-1}$, the quantitative analysis model of PLS for methanol content was established using TQ Analyst software in this spectral range. The RMSE between the measured value of this system and the predicted value of this PLS model is 0.263 , the measurement results of these two methods are shown in Table 4.

TABLE IV. THE COMPARISON OF MEASUREMENT RESULTS BETWEEN THIS SYSTEM AND PLS MODELING METHOD

\begin{tabular}{ccc}
\hline \multirow{2}{*}{ No. } & \multicolumn{2}{c}{ Measurement of methanol content $\%(\mathbf{v} / \mathbf{v})$} \\
\cline { 2 - 3 } & This system & PLS \\
\hline 1 & 0.47 & 0.00 \\
2 & 2.36 & 2.01 \\
3 & 4.22 & 4.03 \\
4 & 5.45 & 5.94 \\
5 & 7.54 & 8.05 \\
6 & 9.69 & 10.02 \\
\hline
\end{tabular}

\section{CONCLUSIONS}

The rapid measurement technology presented in this paper is effective and reliable with the single-wavelength NIR transmission measurement system. The measurement of methanol content only needs to centrifuge the solution sample for 2 minutes, then, the results of methanol content can be obtained within a few seconds by this system. The measurement time of each sample is within 3 minutes, without any other pretreatment and the sample can be recycled completely. Compared to the longtime of synthesis of biodiesel (generally more than $10 \mathrm{~h}$ ), the measurement time satisfies the requirements for process measurement. The experimental results verified the feasibility and accuracy of this method. When using this reaction to research the catalytic efficiency of different types of immobilized lipase, this method can be used to measure the methanol content during synthesis of biodiesel.

Measurement errors can be caused by the methanol volatilization and slight absorbance difference between oleic acid and methyl oleate at $1550 \mathrm{~nm}$ wavelength. But the accuracy will be improved with the increase of methanol content during biodiesel synthesis. It is useful and significant to do the further research.

\section{REFERENCES}

[1] A. Demirbas, A. Bafail, W. Ahmad, and M. Sheikh, "Biodiesel production from non-edible plant oils," Energy Exploration \& Exploitation, vol. 34, issue 2, pp. 290-318, 2016.

[2] M. Anwar, M.G. Rasul, N. Ashwath. "Optimization of biodiesel production process from papaya (Carica papaya) seed oil,” IEEE
International Conference on Power and Energy Systems, pp. 131-134, 2017.

[3] A. Gorji, "A review on the biodiesel production, key parameters in transesterification reaction, its effects on the environment and human health," Journal of Biodiversity \& Environmental Sciences, vol.7, issue 3, pp. 150-185, 2015.

[4] C.Y. Yu, L.Y. Huang, I. Kuan, and S.L. Lee, "Optimized Production of Biodiesel from Waste Cooking Oil by Lipase Immobilized on Magnetic Nanoparticles," International Journal of Molecular Sciences, vol. 14, issue 12, pp. 24074-24086, 2013.

[5] X. Zhao, F. Qi, C. Yuan, W. Du, and D. Liu, "Lipase-catalyzed process for biodiesel production: Enzyme immobilization, process simulation and optimization," Renewable \& Sustainable Energy Reviews, vol. 44, pp. 182-197, 2015.

[6] Y. Liu and X. Hua, "Production of Biodiesel Using a Nanoscaled Immobilized Lipase as the Catalyst," Catalysis Letters, vol. 144, issue 2, pp. 248-251, 2014.

[7] L.P. Christopher, H. Kumar, and V.P. Zambare, "Enzymatic biodiesel: challenges and opportunities," Applied Energy, vol. 119, issue 12, pp. 497-520, 2014.

[8] A. Bajaj, P. Lohan, P.N. Jha and R. Mehrotra, "Biodiesel production through lipase catalyzed transesterification: An overview," Journal of Molecular Catalysis B Enzymatic, vol. 62, issue 1, pp. 9-14, 2010.

[9] R.X. Zhang, D.L. Zhang, and T.Q. Guo, "Comparison of Several Measurement Methods of Methanol Content," Liquor-Making Science \& Technology, vol. 30, issue 4, pp. 111-113, 2009.

[10] L. Wang, and Y.H. Yang, "Optimization of Conditions for Measuring Methanol in Liquor by Fuchsin Sulfurous Acid Method," Contemporary Chemical Industry, vol. 44, issue 8, pp. 1987-1989, 2015.

[11] C. Li, and C. Kong, "Determination of Methanol Content in Biodiesel by Headspace Gas Chromatography," Petrochemical Technology, vol. 41, issue 10, pp. 1196-1200, 2012.

[12] M. C. Paraschivescu, E.G. Alley, W.T. French, R. Hernandez, and K. Armbrust. "Determination of methanol in biodiesel by headspace solid phase microextraction," Bioresource Technology, vol. 99, issue 13, pp. 5901-5905, 2008.

[13] A.G. Ouyang, Z.H. Huang, and Y.D. Liu, "Determination of Methanol Content in Methanol Diesel Based on Near Infrared Spectroscopy," Spectroscopy \& Spectral Analysis, vol. 37, issue 4, pp. 1118-1122, 2017

[14] J. Kang, W. Cai, and X. Shao, "Quantitative determination by temperature dependent near-infrared spectra: a further study," Talanta, vol. 82, issue 3, pp. 1017-1021, 2010.

[15] X.M. Wu, J.Y. Xin, Y.X. Zhang, Z.D. Zhai, and C.G. Xia, "Advances in Lipase-catalyzed Reaction in Solvent-free Condition," Journal of Molecular Catalysis, vol. 20, issue 6, pp. 597-603, 2006.

[16] S. Wu, Y. Liu, Q. Li, and M. Shi, "Lipase-catalyzed preparation of biodiesel from jatropha oil in solvent-free system," Chemical Reaction Engineering \& Technology, vol. 27, issue 4, pp. 322-326, 2011.

[17] N. Choi, J.S. Lee, J. Kwak, J. Lee, and I.H. Kim, "Production of Biodiesel from Acid Oil via a Two-Step Enzymatic Transesterification," Journal of Oleo Science, vol. 65, issue 11, pp. 913-921, 2016. 